\title{
Heritability of a skeletal biomarker of biological aging
}

\author{
Ida Malkin · Leonid Kalichman • \\ Eugene Kobyliansky
}

Received: 15 March 2007 / Accepted: 25 May 2007/Published online: 23 June 2007

(C) Springer Science+Business Media B.V. 2007

\begin{abstract}
Changes in the skeletal system, which include age-related bone and joint remodeling, can potentially be used as a biomarker of biological aging. The aim of the present study was to investigate the extent and mode of inheritance of skeletal biomarker of biological aging-osseographic score (OSS), in a large sample of ethnically homogeneous pedigrees. The investigated cohort comprised 359 Chuvashian families and included 787 men aged 18-89 years (mean 46.9) and 723 women aged 18-90 years (mean 48.5). The TOSS - transformed OSS standardized in 5-year age groups for each sex, was analyzed as a BA index. We evaluated familial correlations and performed segregation analysis. Results of our study suggest the familial aggregations of TOSS variation in the Chuvashian pedigrees. In a segregation analysis we found a significant major
\end{abstract}

I. Malkin $(\bowtie) \cdot$ E. Kobyliansky

Human Population Biology Research Unit, Department of Anatomy and Anthropology, Sackler Faculty of Medicine, Tel Aviv University, Ramat Aviv, Tel Aviv 69978, Israel e-mail: idak@post.tau.ac.il

L. Kalichman $(\varangle)$

Department of Physical Therapy, The Stanley Steyer

School of Health Professions, Sackler Faculty of

Medicine, Tel Aviv University, Tel Aviv, Israel

e-mail: kalihman@zahav.net.il

E. Kobyliansky

Lilian and Marcel Pollak Chair of Biological

Anthropology, Sackler Faculty of Medicine,

Tel Aviv University, Tel Aviv, Israel gene (MG) effect in the individual's TOSS with a dominant most parsimonious model $\left(\mathrm{H}^{2}=0.32\right)$. Genetic factors (MG genotypes) explained $47 \%$ of the residual OSS variance after age adjustment and after including sex-genotype interaction, they explained $52 \%$ of the residual variance. Results of our study also indicated that the inherited difference in the skeletal aging pattern in men lies mostly in the rate of aging, but in women in the age of the onset of the period of visible skeletal changes.

Keywords Osseographic score - Skeletal aging · Chuvashia $\cdot$ Biological aging $\cdot$ Heritability

\section{Introduction}

Biological age (BA) estimates the functional status of an individual in reference to his or her chronological peers on the basis of how well he or she functions in comparison with others of the same chronological age (Borkan and Norris 1986). Changes in the skeletal system, which include age-related bone and joint remodeling, can potentially be used as a biomarker of BA. Skeletal aging measures are strongly correlated with the status of the vital health systems (Gabriel et al. 1999; Kiel et al. 2001, Kadam et al. 2004, Kalichman et al. 2006b) and ultimately with survival rates (Johansson et al. 1998; Haara et al. 2003). The rate of degenerative changes in the skeleton may reflect an individual's biological resistance, 
immunity, functional or health status in reference to his or her chronological age. Therefore, age-related skeletal changes can possibly be used as an index of BA.

Roentgenography is one of the common methods used to assess health status and age-related changes in bones and joints (Plato 1987; Plato et al. 1994). Previously, Kobyliansky et al. (1995) reported an osseographic scoring (OSS) system that uses hand radiographs to assess skeletal aging. OSS is a synthetic measure for assessing skeletal aging based on radiographic features of the hand, combining both osteoporotic and osteoarthritic changes of hand bones and joints. This system was originally suggested by Pavlovsky (1987) and has been used in BA evaluations in population studies (Kobyliansky et al.1995; Livshits et al. 1996; Pavlovsky and Kobyliansky 1997). Importantly, OSS has been shown to highly correlate with chronological age in adults of different ethnic groups (Karasik et al. 1999, Kalichman et al. 2002).

Accumulating research shows that single gene changes in yeast, worms, and rodents can significantly alter life span (Kenyon et al. 1993; Kaeberlein et al. 1999; Cheng et al. 2003; McBurney et al. 2003). The observation that the first-degree relatives of human centenarians are both disease resistant and have longer than average life spans implies a genetic component to human longevity (Perls et al. 2002; Atzmon et al. 2004). Current estimates based on twin studies indicate that the human life span is $25 \%$ heritable (McGue et al. 1993; Herskind et al. 1996; Gudmundsson et al. 2000; Perls et al. 2002). Genetic factors may also significantly contribute to interindividual differences in BA (Karasik et al. 2004, 2005). In variance component genetic analysis, performed in Karasik's study (2004), genetic factors explained $57 \%$ of the total variance of the BA index based on OSS measurements.

The aim of our study was to investigate the extent and mode of inheritance of a skeletal biomarker of biological aging (OSS) in a large sample of ethnically homogeneous pedigrees.

\section{Materials and methods}

\section{Sample}

The population sampled consisted of native Chuvashians residing in numerous small villages in the
Chuvashia and Bashkartostan Autonomies of the Russian Federation. The data were gathered in three field studies during August/September of 1994 (555 individuals), May/June of 1999 (715 individuals), and September 2002 (240 individuals) by investigators from the Department of Anatomy and Anthropology, Sackler Faculty of Medicine, Tel Aviv University (Israel), and the Institute of Anthropology, Moscow University (Russia). The expeditions were part of a Chuvashia Skeletal Aging Study (ChuSAS) project whose main aim was to investigate the different aspects of skeletal aging within the Chuvashia population. All information collected and measurements taken during all expeditions were done by the same team of investigators. The investigated cohort comprised 359 families and included 787 men aged 18-89 years (mean 46.9) and 723 women aged 18-90 years (mean 48.5).

The Chuvashian population is characterized by a stable family structure with traditional relationships. For generations the Chuvashians have resided under the same environmental conditions and have not been exposed to an outside genetic flow (El'chinova et al. 2003). In the present study, we collected data from residents of a number of small villages (with a predominant Chuvashian population) located in the Volga region. A rural population is more homogeneous than urban in terms of ethnicity, occupation, and physical activity. The study participants shared similar living, economic, and professional conditions with most individuals employed in agriculture or in other occupations involving physical labor. Data from $80 \%$ to $90 \%$ of the families (including all family members who were living in the area at the time of the expedition) were obtained. Since almost every individual was related to one of the families, we were able to collect data on up to $90 \%$ of the population in each village. Data obtained from selfcompleted questionnaires and confirmed during the interview included sex, age, age at menarche, age at menopause and occupation. Menopause was defined as $>12$ months without menstruation. Age at last menstruation was considered as menopausal age. In order to minimize the possible recall bias, the investigators excluded from the analyses all women who had doubts about their age at menarche. The anthropometrical measurements (weight, stature, etc.) were also collected using standard methodology. In the studied sample, there were no individuals with 
known rheumatoid or psoriatic arthritis or users of steroid medication. Subjects with post-traumatic hand osteoarthritis were excluded from the study. All procedures involved were consensual. The subjects signed an informed consent form, and the entire project was approved by the Helsinki Ethics Committee of Tel-Aviv University.

Additional information about the studied population can be found in our previous publications: sexand age-related variations of the somatotype (Kalichman and Kobyliansky 2006), reproductive indices, such as age at menarche and at menopause (Kalichman et al. 2006 a, 2007a) and sex- and agerelated variations of OSS (Kalichman et al. 2007b).

\section{Hand radiographs}

Single plain radiograms of both hands were taken from each study participant in the postero-anterior position with the X-ray source located $60 \mathrm{~cm}$ above, using a standard radiographic technique, as described in detail by Livshits et al. (1996). Hands were placed on the same film-containing plate to avoid any film or development variation. It was exposed for 5-10 s at $100-150 \mathrm{~mA}$ without intensifying screens at $50 \mathrm{kV}$.

\section{OSS evaluation}

An individual's OSS was determined by the occurrence of the following: (a) bone proliferations (spurs), including apiostoses (tufting of distal phalangeal tuberosity), osteophytes, enthesophytes of the juxtaarticular area and at midshaft; (b) manifestations of bone porosity, such as resorption of trabeculae, development of lacuna (juxta-articular osteopenia) and erosion of cortex; (c) loci of osteosclerosis, defined as enostosis and sclerotic nuclei; and subchondral sclerosis; and (d) non-traumatic joint deformities, defined as narrowing of joint cavities and periarticular bone corrosion (Kobyliansky et al. 1995; Pavlovsky and Kobyliansky 1997; Karasik et al. 2005; Kalichman et al. 2007b). Phalanges of the 2nd to 5th fingers of the left hand were examined. The first finger (thumb) does not directly project in a standard X-ray and was therefore not utilized. The presence of a given feature, but not its level of development, was documented for each OSS estimate. The overall number of those skeletal aging features was used as an OSS.
Each radiograph was read by an experienced skeletal researcher according to the accepted protocol of OSS evaluation. Ten X-rays were read and then reread by the investigator to estimate the intra-observer reliability of the readings. All discrepancies were reviewed for systematic errors. This exercise continued until the reliability was high $(k>0.8)$. Afterwards, the investigator read the remaining X-rays, still blinded to patient identifiers. Before reading each batch of X-rays one re-read $5 \mathrm{X}$-rays, which had been previously read, to "calibrate" one's readings to a standard. The intra-observer reliability (kappa statistics) was $0.82(P<0.01)$. The intraclass correlation for the OSS was $0.92(P<0.01)$.

OSS reflects amount of skeletal changes occurring stochastically during the life-span, and therefore it is characterized by strong increase of trait variance with age (Kalichman et al. 2007b and Fig. 1). It is reasonable to assume that not the deviation of OSS from the age-group mean, but only relative rate of OSS accumulation with age can be inherited. Consequently we constructed a trait using the standardization in age-sex groups to equalize the means and variances in the whole age range for both sexes. The resulting trait, TOSS (transformed OSS) was further analyzed. The biological meaning of this trait is the relative position of individual (higher or lower than average values of OSS) in his/her age-sex group expressed in the units of SD specific for each group. This accurately fit the definition of BA as the functional status of an individual in reference to his or her chronological peers (Borkan and Norris 1986).

Statistical and genetic analysis

Prior to any other evaluations, the descriptive statistics reflecting the basic characteristics of the studied sample were computed in the whole sample and within age groups of 5 years.

At the first stage we computed familial (spouses, parent-offspring and sib-sib) correlations between the corresponding TOSS levels to examine the potential genetic effect on the skeletal biomarker of BA.

The segregation analysis, as implemented in the statistical package MAN (Malkin and Ginsburg 2003), was utilized in this study to test a mode of inheritance of the skeletal biomarker of BA-TOSS. Only individuals older than 29 were included in the analysis, since the high prevalence of zero OSS 
Fig. 1 Mean OSS values and standard deviations (SD) in age groups of 5 years for men and women

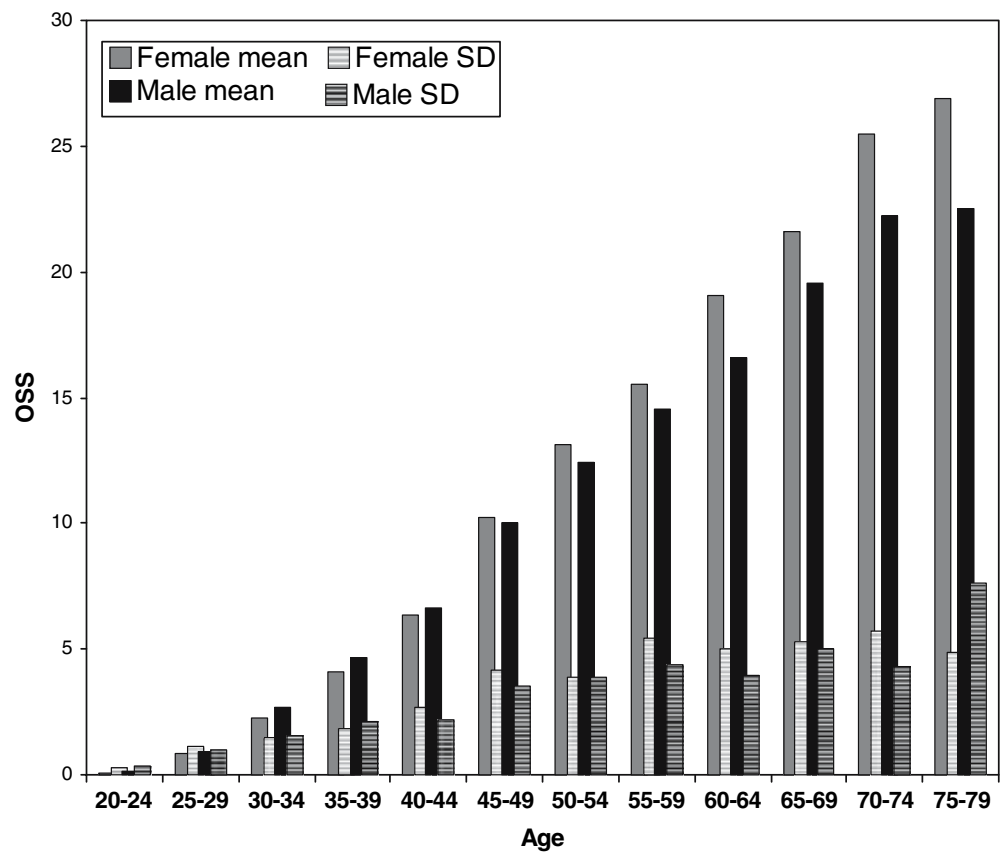

values in younger individuals make the standardization approach unacceptable. More precisely, in two 5-year age groups younger than 30 years the distribution of TOSS significantly deviated from normal (Chi-square $\chi^{2}$-test for frequencies gave $P<10^{-6}$ ), whereas in all older age groups did not $(P>0.01)$. Only pedigrees having at least one measured pair of relatives were included in the analysis. The mixed model of inheritance (Morton and MacLean 1974; Elston 1981; Lalouel 1983; Beaty 1997; Ginsburg and Livshits 1999) estimates the effects of a potential major gene and possible multifactorial effects, and is described in detail in numerous publications, including the aforementioned. The parameters that had been estimated in the general model are defined as follows: $\mathrm{p}$ - the population frequency of allele $\mathrm{A}_{1}$ (assuming diallelic gene locus); $\mu_{\mathrm{gs}}$ - the average genotypic value of individuals having genotype $g$ and sex $s$ ( $g=1,2$, and 3 corresponds to genotypes $\mathrm{A}_{1} \mathrm{~A}_{1}$, $\mathrm{A}_{1} \mathrm{~A}_{2}$, and $\mathrm{A}_{2} \mathrm{~A}_{2}$, respectively); $\sigma_{\mathrm{g}}^{2}$ - the trait variance in individuals having the same major gene genotype $g$, it estimates the trait variation resulting from the effect of all possible environmental factors and potential minor genes; $\tau_{\mathrm{g}}$-the transmission probability parameter, which estimates the probability that a parent of genotype $g$ transmits allele $\mathrm{A}_{1}$ to the next generation; $\rho, \beta$, and $\varepsilon$ are partial correlations between the trait's residuals adjusted for a major gene effect, in spouses, parents/offspring, and the siblings, respectively. A transmission probability test, described by Ginsburg and Livshits (1999), was used to estimate the significance of the major gene (MG) effect. A best-fitting and most parsimonious Mendelian model was established after dropping all nonsignificant parameters.

Using the procedure implemented in the package MAN (Malkin and Ginsburg 2003) and parameters estimated for the most parsimonious model, we determined the most likely MG genotype combination for each pedigree. We divided our sample into groups according to sex and the determined individual genotype of putative BA-related MG for genotypes having distinguishable genotypic values.

To analyze the age dependence of OSS, as a primary (directly measured) BA-related index, in groups of potentially different inherited BA, we used the stochastic model, described in detail by Kobyliansky et al. (1995). We estimated for each group the following aging population parameters: $t_{0}$ - the earliest age at which the first signs of OSS can be found, $q$-the individual probability of revealing the first signs of OSS changes per chronological time unit, assuming an individual's age $\geq t_{0}$, and $B$-the coefficient characterizing the rate of OSS changes 
per time unit (per year) after the process of aging has started. Next, we built the age-dependent expectation of the mean OSS level for each group according to sex and predicted MG genotype. Using the likelihood ratio test, we compared the extent of stochastic model approximation, both accounting for the MG genotype group and without it. Eventually we used ANOVA as implemented in STATISTICA 6.0 (StatSoft 2000) to test the differences between parameters of female organism development such as age at menarche and age at menopause in MG genotype groups.

\section{Results}

Figure 1 shows the OSS mean values and SD in age groups of 5 years. Up to age 45, men have higher OSS scores than women. After age 45 OSS in women was higher, compared with men and the sex difference in the OSS scores increases up to the end of the age range. The OSS variance in age groups increases rapidly up to age 50 .

Familial correlations of TOSS (Table 1) showed no significant correlation for spouses, but parentoffspring and sibling correlations were significant. This indicates the existence of a clear familial aggregation of TOSS variation in the Chuvashian pedigrees, which cannot be explained by pure common environmental effects. We investigated the nature of TOSS inheritance later using segregation analysis. Because of the method by which TOSS was constructed (standardization within each sex-age group), in the segregation model the sex effects were not significant. The other results are presented in Table 2 and support the MG hypothesis regarding trait inheritance. The most parsimonious Mendelian model was dominant. The recessive allele with a population frequency of 0.6 corresponded to relatively slow aging. For the most parsimonious Mendelian model, $\mathrm{H}^{2}=0.315$; total proportion of TOSS variance explained by the model was 0.349 . Using

Table 1 Familial correlations of TOSS

\begin{tabular}{llll}
\hline Relatives & $R$ & $N$ of pairs & $P$ \\
\hline Spouses & 0.08 & 333 & 0.15 \\
Parent-offspring & 0.12 & 755 & 0.002 \\
Sib pairs & 0.31 & 189 & 0.0008 \\
\hline
\end{tabular}

this model, we predicted the most likely MG genotype combination for each pedigree included in the analysis. Because of the dominant nature of the most parsimonious model, we divided all individuals into two groups with potentially different genotypic values: (1) homozygous on allele $A_{1}$ and (2) all the rest. Using a stochastic model of aging (Kobyliansky et al. 1995), we constructed the model of age dependence of directly measured OSS for a whole sample and for each predicted genotype group separately with and without considering the effects of sex. Table 3 presents the parameters of four resulting models and a comparison between them. According to the likelihood ratio test (LRT), Model 1, which included only the effect of age, has a significantly worse extent of approximation than both Model 2, which included the effect of genotype-age, and Model 3, which included the effect of sex-age. The extent of approximation was significantly higher for the effect of genotype-age, which explained $47 \%$ of the residual OSS variance after only adjustment for age, and lower for the effect of sex-age (5\%). Model 4, which includes all age-sex-genotype effects, showed a significantly higher extent of approximation in comparison with other models and explained 52\% of the residual OSS variance after adjustment for age. After adjustment for age and sex, the residual parentoffspring correlation of OSS was significant, but became non-significant after adjustment for the sexage-genotype.

Figure 2 shows a scatterplot of OSS-age for the predicted genotypes in both sexes and the corresponding curves of age-dependent OSS expectation given by the stochastic model. For women in the group of genotype $A_{1} A_{1}$ (with slow skeletal aging), the age at menarche was significantly higher $(P=0.012)$ than in the group of genotypes $\mathrm{A}_{1} \mathrm{~A}_{2}$ or $\mathrm{A}_{2} \mathrm{~A}_{2}$; the group mean difference was 0.45 year. The difference in the age at menopause was in the same direction, but not significant for the whole sample. Note that our sample includes women strongly influenced by difficult life conditions during World War II. In these women the variance in the age at menopause is increased in comparison with the normal population. For women whose year of birth $\geq 1937$ (the normal part of the population), the age at menopause was higher in group $\mathrm{A}_{1} \mathrm{~A}_{1}$ than in other group $(P=0.054)$; the group mean difference was 1.6 year. 
Table 2 Segregation analysis for TOSS (OSS standardized in age groups of 5 years for each sex)

\begin{tabular}{|c|c|c|c|c|c|c|}
\hline \multirow[t]{2}{*}{ Parameter } & \multicolumn{3}{|c|}{ Main models } & \multicolumn{3}{|c|}{ Restricted models } \\
\hline & $\begin{array}{l}\text { General } \\
1\end{array}$ & $\begin{array}{l}\text { Mendelian } \\
2\end{array}$ & $\begin{array}{l}\text { Equal } \tau \text {-s } \\
3\end{array}$ & $\begin{array}{l}\text { Arbitrary } \tau \text {-s } \\
4\end{array}$ & $\begin{array}{l}\text { Mendelian most parsimonious } \\
5\end{array}$ & $\begin{array}{l}\text { Equal } \tau \text {-s } \\
6\end{array}$ \\
\hline$P$ & 0.600 & 0.628 & 0.252 & 0.558 & $0.598 \pm 0.033$ & 0.563 \\
\hline$\mu_{1}$ & -0.727 & -0.724 & -1.080 & -0.758 & $-0.729 \pm 0.057$ & -0.702 \\
\hline$\mu_{2}$ & 0.481 & 0.525 & -0.329 & 0.380 & $0.429 \pm 0.061$ & 0.362 \\
\hline$\mu_{3}$ & 0.362 & 0.385 & 0.383 & $0.380 !$ & $0.429 !$ & $0.362 !$ \\
\hline$\sigma_{1}^{2}$ & 0.299 & 0.325 & 0.074 & 0.255 & $0.303 \pm 0.045$ & 0.289 \\
\hline$\sigma_{2}^{2}$ & 1.074 & 0.988 & 0.504 & 1.165 & $1.067 \pm 0.103$ & 1.181 \\
\hline$\sigma_{3}^{2}$ & 0.205 & 0.232 & 1.049 & $0.255 !$ & $0.303 !$ & $0.289 !$ \\
\hline$\rho$ & 0.085 & 0.104 & 0.099 & {$[0.000]$} & {$[0.000]$} & {$[0.000]$} \\
\hline$\beta$ & 0.006 & -0.029 & 0.033 & {$[0.000]$} & {$[0.000]$} & {$[0.000]$} \\
\hline$\varepsilon$ & 0.325 & 0.228 & 0.311 & 0.327 & $0.249 \pm 0.067$ & 0.327 \\
\hline$\tau_{1}$ & 0.910 & {$[1.000]$} & 0.272 & 0.916 & {$[1.000]$} & 0.614 \\
\hline$\tau_{2}$ & 0.632 & {$[0.500]$} & $0.272 !$ & 0.618 & {$[0.500]$} & $0.614 !$ \\
\hline$\tau_{3}$ & 0.001 & {$[0.000]$} & $0.272 !$ & 0.020 & {$[0.000]$} & $0.614 !$ \\
\hline Log LH & -1425.66 & -1428.27 & -1435.91 & -1427.29 & -1429.61 & -1440.51 \\
\hline$\chi^{2}$ & - & $5.22^{\mathrm{NS}}(1)$ & $20.5^{* *}(1)$ & $1.63^{\mathrm{NS}}(1)$ & $4.64^{\mathrm{NS}}(4)$ & $26.44 * *(4)$ \\
\hline
\end{tabular}

NS-P>0.05; ** $P<0.01$

Number in parentheses in the $\chi^{2}$-row specifies a comparative column

Constraints denotation: [ ] - parameter is fixed to shown value; !- parameter is constrained to be equal to the parameter above in the table

To compare our findings with results of other authors, we quantitatively analyzed the similarity of differently constructed BA indices. The correlation between the BA index proposed by Karasik et al. (2004) and TOSS was 0.82 in the Chuvashian population. The correlations between the BA index, used by Livshits et al. (1996), versus the BA of Karasik et al. (2004) and TOSS were estimated as 0.80 and 0.89 , correspondingly. Though the resemblance of the indices may be influenced by population type, the aforementioned BA indices are sufficiently similar.

\section{Discussion}

In the present study, we evaluated the extent and mode of inheritance of a skeletal biomarker of BA. We constructed this biomarker (TOSS) to be maximally fitted according to the BA definition; it reflects the intensity of an individual's skeletal changes in relation to its coevals of the same sex, and the range of the index does not depend on the age cohort.
We previously evaluated the association between OSS, as an index of BA, with morbidity in a general Chuvasha population (Kalichman et al. 2006b). Statistically significant association was found in the present study between age-adjusted OSS and rheumatic morbidity. Morbidities such as ischemic heart disease, pulmonary diseases, traumatic brain injuries and gynecological diseases also showed differences between mean values of OSS in affected vs. non-affected individuals. However, after correction for multiple testing, the statistical significance was insufficient. The association between OSS and diabetes mellitus was close to significant $(P=0.07)$ and we surmise that with a larger sample of diabetic individuals, a clear-cut association will be confirmed.

It is well-established that genetic factors play an important role in an individual's BA (Karasik et al. 2005). Our finding suggests the familial aggregations of TOSS variation within the Chuvashian pedigrees. The evaluation of heritability of the skeletal biomarker of BA in segregation analysis suggested the existence of a MG effect with a most parsimonious 
Table 3 Parameters of stochastic aging models estimated for OSS data without grouping (1) and grouped according to predicted genotype (2), sex (3), or both sex and predicted genotype (4)

\begin{tabular}{|c|c|c|c|c|}
\hline & Parameter & All & $\mathrm{A}_{1} \mathrm{~A}_{1}$ & $\mathrm{~A}_{1} \mathrm{~A}_{2}$ or $\mathrm{A}_{2} \mathrm{~A}_{2}$ \\
\hline \multirow[t]{8}{*}{ All } & $t_{0}$ & $21.223 \pm 0.933$ & $21.705 \pm 0.884$ & $22.225 \pm 1.179$ \\
\hline & $q$ & $0.118 \pm 0.015$ & $0.077 \pm 0.010$ & $0.217 \pm 0.044$ \\
\hline & $\mathrm{B}$ & $0.552 \pm 0.004$ & $0.518 \pm 0.004$ & $0.600 \pm 0.004$ \\
\hline & \multirow[t]{5}{*}{$\mathrm{TM}$} & 29.689 & 34.619 & 26.838 \\
\hline & & Model 1 (3 d.f.) & Model 2 (6 d.f.) & \\
\hline & & $R^{2}=0.838$ & $R^{2}=0.915$ & \\
\hline & & $\log \mathrm{LH}=-3476.8$ & $\log \mathrm{LH}=-3065.5$ & \\
\hline & & - & $\chi^{2}=822.6$ & \\
\hline \multirow[t]{4}{*}{ Men } & $\mathrm{t}_{0}$ & $20.524 \pm 1.231$ & $21.573 \pm 1.142$ & $21.886 \pm 1.683$ \\
\hline & $q$ & $0.127 \pm 0.024$ & $0.098 \pm 0.014$ & $0.233 \pm 0.071$ \\
\hline & $\mathrm{B}$ & $0.501 \pm 0.005$ & $0.449 \pm 0.005$ & $0.556 \pm 0.005$ \\
\hline & $\mathrm{TM}$ & 28.400 & 31.740 & 26.182 \\
\hline \multirow[t]{8}{*}{ Women } & $\mathrm{t}_{0}$ & $22.078 \pm 1.342$ & $21.840 \pm 1.169$ & $22.623 \pm 1.601$ \\
\hline & $q$ & $0.107 \pm 0.019$ & $0.057 \pm 0.000$ & $0.187 \pm 0.046$ \\
\hline & $\mathrm{B}$ & $0.617 \pm 0.007$ & $0.629 \pm 0.008$ & $0.657 \pm 0.007$ \\
\hline & \multirow[t]{5}{*}{$\mathrm{TM}$} & 31.400 & 39.373 & 27.979 \\
\hline & & Model 3 (6 d.f.) & \multicolumn{2}{|l|}{ Model 4 (12 d.f.) } \\
\hline & & $R^{2}=0.846$ & \multicolumn{2}{|l|}{$R^{2}=0.923$} \\
\hline & & $\log \mathrm{LH}=-3443.3$ & \multicolumn{2}{|l|}{$\log \mathrm{LH}=-3000.7$} \\
\hline & & $\chi^{2}=67.0$ & \multicolumn{2}{|c|}{$\chi^{2}=952.2(1) ; 129.6(2) ; 885.2(3) ;$} \\
\hline
\end{tabular}

$\mathrm{A}_{1} \mathrm{~A}_{1}$ - genotype corresponding to lower values of TOSS in segregation analysis; $t_{0}$ - the minimal age at which initial bone changes occurred in a group; $q$ - the group probability that an individual will first develop involutive bone change after age $t_{0} ; B$ - the group rate of aging (bone change) per time unit; TM - the expected mean age in a group in which the first bone change occurred; number in parentheses indicates the comparative model for LRT; all LRT values are significant with $P<10^{-6}$

Fig. 2 OSS values for predicted genotype groups: black- $A_{1} A_{1}$, gray- $A_{1} A_{2}$ or $\mathrm{A}_{2} \mathrm{~A}_{2}$
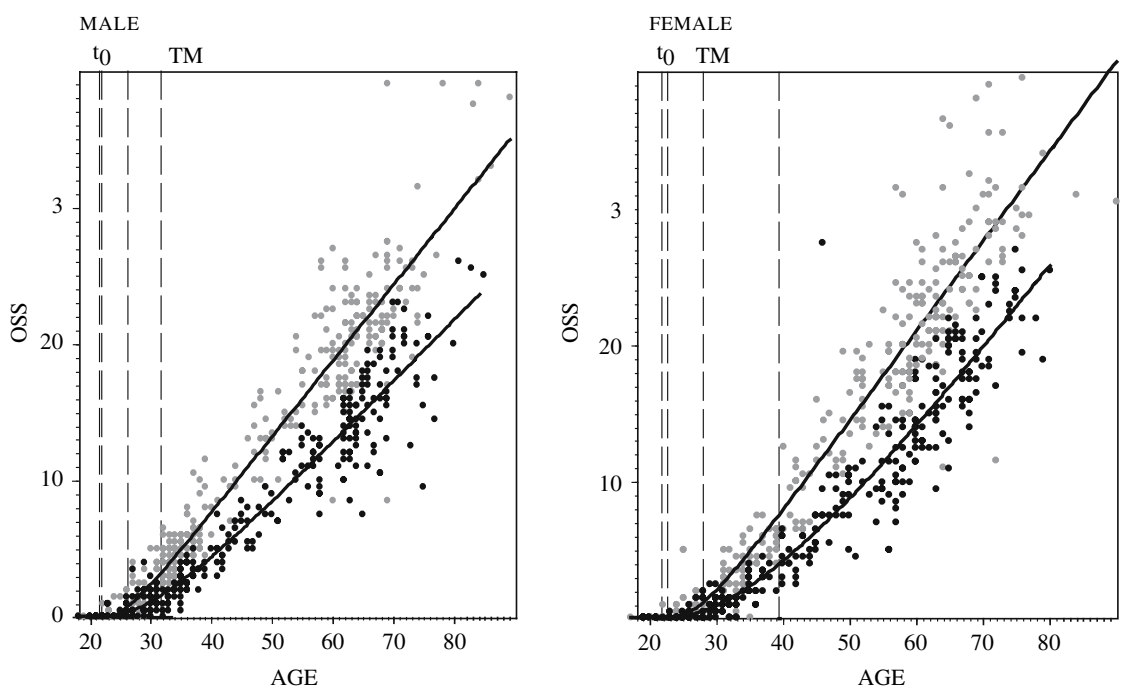

dominant model. A number of BA indices, based on age-related skeletal changes, were previously proposed and analyzed in different populations. Livshits et al. (1996) analyzed the OSS measurement in the Turkmenian population. They used the age-adjusted OSS values as a BA index. This index showed a 
significant familial parent-offspring correlation of $0.1 \pm 0.09$, which is approximately the same as for TOSS in our study (0.12). Nevertheless, in their segregation analysis of the age-adjusted OSS, the Mendelian model was rejected. A possible reason is that a simple age adjustment does not equalize the residual variance of OSS, which significantly increases with age. As a result, parents and offspring received a BA index with different amplitude and this difference probably prevented establishing MG inheritance in segregation analysis. Other phenotypes of BA, as well, are characterized by substantial heritability. Thus, functional age (other term for BA) has been assessed by a general linear regression model of many biomarkers in Minnesota twins (27-88 years old). A subset of these biomarkers (mostly physiological variables) identified by a factor analysis had heritability of $\sim 59 \%$ (Finkel et al. 1995). Duggirala et al. (2002) have reported that BA, measured according to Uttley and Crawford (1994), had a substantial genetic component (heritability ranging from $0.27 \pm 0.11$ in one Mennonite geographical subsample to $0.47 \pm 0.18$ in the other).

Karasik et al. (2004) studied a sample that included 1,402 members of 288 pedigrees from the Framingham Heart Study; the log-transformation was used to reduce the growth of OSS variance with age. A special BA index was constructed by adjusting the individual's age for log-transformed OSS with cubic polynomial. The log-transformation automatically excludes from the analysis all individuals with zero OSS (note that for ages $>30$, the zero OSS value is meaningful and denotes a low BA). For the resulting trait, the genetic variance component analysis showed that sex, height, the body mass index, and, in women, menopausal status and estrogen use, jointly explained approximately $6 \%$ of the total variance of BA index. Genetic factors explained an additional $57 \%$. The genome search for this BA index revealed a number of potential chromosomal regions in the two-point analysis, but for multipoint, only the region on chromosome 21 remained significant.

The variance component analysis, used by Karasik et al. (2004), a priori assumes a polygene mode of inheritance. Our segregation analysis results confirm the findings about a significant genetic effect and furthermore suggest the existence of a MG effect in an individual's TOSS with a dominant most parsimonious model. The MG hypotheses may be, to some extent, supported by the fact that in the multipoint variance component linkage analysis by Karasik et al. (2004) only one chromosomal region showed a significant LOD score $>3$ (likelihood ratio greater than $10^{3}$ ). We found that genetic factors (MG genotypes) explained $47 \%$ of the residual OSS variance after adjustment for age, and after including the sex-genotype interaction, they explained $52 \%$ of the residual variance. For TOSS the MG effect was $32 \%$.

Additionally, we compared the age dependence of OSS by directly measuring the individual's skeletal health index in predicted MG genotype groups. Kobyliansky et al. (1995) proposed a two-stage stochastic statistical model of the population aging pattern for OSS. The implied stages are as follows: (1) the period of life between birth and the apparent onset of skeletal changes $\left(t<t_{\mathrm{i}}\right)$, defined as the latent period, and (2) the period of visible changes beginning after $t_{\mathrm{i}}$ (which is not the same in different individuals), when involutive changes occur continuously throughout the remaining life. Applying this model separately in predicted genotype groups, we compared their aging features by means of model parameters $\left(t_{0}, q, B\right)$. The earliest age of the first visible changes, $t_{0}$, was almost the same in all sexgenotype groups, about 22 years. The probability per year of transition from the latent to the visible changes period, $q$, was for genotype $\mathrm{A}_{1} \mathrm{~A}_{1}$ about $1 / 3$ of probability for other individuals. This was especially expressed for women and it results in greater values of the average transition age TM (39.3 years in $\mathrm{A}_{1} \mathrm{~A}_{1}$-group and 31.7 for the others). The rate of aging, $B$, was also significantly different in the predicted genotype groups, but this difference was expressed more for men. Thus, we can conclude that the inherited difference in the aging pattern lies in men, mostly in the rate of aging, but in women, it is in the age of onset of the period of visible changes.

An additional finding was that in the group $\mathrm{A}_{1} \mathrm{~A}_{1}$ (slow aging), for women, the age at menarche was significantly greater $(P=0.012)$ than in the group $\left\{A_{1} A_{2}\right.$ OR $\left.A_{2} A_{2}\right\}$. For women born after 1937 (those whose maturation period was not influenced by WW II or the subsequent rehabilitation period), the age at menopause was also greater in the group $\mathrm{A}_{1} \mathrm{~A}_{1}$ $(P=0.054)$. Thus, there is a positive relation between the rate of sexual development and the rate of aging, characterized by degenerative skeletal changes. The 
rate of sexual development is positively associated with intensity of normal metabolism, which also may lead to accelerated aging. Early onset of menarche is also known to be linked to other aging conditions such as elevated blood pressure and glucose intolerance, compared with later maturing girls, independent of body composition (Remsberg et al. 2005).

The main molecular characteristic of aging is the progressive accumulation of damages in macromolecules that result on macro level in heterogeneity of tissues and degenerative changes. Rattan (2006) pointed out three major sources of this damage: (1) reactive oxygen species (ROS) and free radicals formed due to external inducers of damage (for example ultra-violet rays), and as a consequence of cellular metabolism; (2) nutritional glucose and its metabolites, and their biochemical interactions with ROS; and (3) spontaneous errors in biochemical processes, such as DNA duplication, transcription, post-transcriptional processing, translation, and posttranslational. Bessenyei et al. (2004) reviewed data concerning SNPs associated with life span positioned in a variety of genes regulating metabolic processes, apoptosis, stress response (see also Singh et al. 2006) and immune response. Several polymorphisms in ERalpha (estrogen receptor that are known to play significant role in skeletal development) were reported to be associated with life span (Bessenyei et al. 2004) and with both osteoarthritis (Jin et al. 2004) and osteoporosis (Albagha et al. 2005) related phenotypes. The VDR (vitamin D receptor) gene is also known to be associated with both types of degenerative changes in certain skeletal sites (Uitterlinden et al. 1997; Yue et al. 2005). A great number of genes with significant association signals were cited in recent reviews of Loughlin (2005) (osteoarthritis) and Liu et al. (2006) (osteoporosis). de Boer et al. (2002) described studies of mice with a mutation in XPD, a gene encoding a DNA helicase that functions in both repair and transcription. Mice with mutation were found to exhibit many symptoms of premature aging, including osteoporosis and kyphosis, osteosclerosis, early greying, cachexia, infertility, and reduced life-span. So OSS, the index comprising various accumulated degenerative skeletal changes, despite to different molecular mechanisms, which are involved in bone loss, cartilage loss and other OSS components, may be under genetic control of some polymorphic genes taking part in
DNA repair pathways, transcription and translation control and/or some polymorphic genes controlling intensity of normal metabolism, that influence the rate of molecular damage (Kirkwood and Austad 2000). This supports the assumption that OSS as a skeletal biomarker of BA can be used to evaluate the organism's general aging status.

Acknowledgments Grants: This study was supported by Grant No. 1042-04 from the Israel National Science Foundation, and by Grant No. 900010 from Tel Aviv University.

\section{References}

Albagha OME, Pettersson U, Stewart A et al (2005) Association of oestrogen receptor gene polymorphisms with postmenopausal bone loss, bone mass, and quantitative ultrasound properties of bone. J Med Genet 42:240-246

Atzmon G, Schechter C, Greiner W et al (2004) Clinical phenotype of families with longevity. J Am Geriatr Soc 52:274-277

Beaty TH (1997) Evolving methods in genetic epidemiology. I. Analysis of genetic and environmental factors in family studies. Epidem Rev 19:14-23

Bessenyei B, Màrka M, Urbàn L et al (2004) Single nucleotide polymorphisms: aging and diseases. Biogerontology 5:291-303

Borkan GA, Norris AH (1986) Assessment of biological age using a profile of physical parameters. J Gerontol 35:177184

Cheng HL, Mostoslavsky R, Saito S et al (2003) Developmental defects and p53 hyperacetylation in Sir2 homolog (SIRT1)-deficient mice. Proc Natl Acad Sci USA 100:10794-10799

Dean W, Morgan RF. (1988) In defense of the concept of biological aging measurement-current status. Arch Gerontol Geriatr 7:191-210

de Boer J, Andressoo JO, de Wit J et al (2002) Premature aging in mice deficient in DNA repair and transcription. Science 296:1276-1279

Duggirala R, Uttley M, Willams K et al (2002) Genetic determination of biological age in the Mennonites of the Midwestern United States. Genet Epidemiol 23:97-109

El'chinova GI, Znichenko RA, Ginter EK (2003) Time Dynamics of Ethnic Assortativeness in Tsivil'sk Raion of Chuvashia. Rus J Genetics 39:461-462

Elston RC (1981) Segregation analysis. In: Mielke JH, Crawford $\mathrm{MH}$ (eds) Current developments in anthropometrical genetics, vol I. Plenum Press, New York, pp 327-354

Finkel D, Whitfield K, McGue M (1995) Genetic and environmental influences on functional age: a twin study. J Gerontol B Psychol Sci Soc Sci 50:P104-P113

Gabriel SE, Crowson CS, O'Fallon WM (1999) Comorbidity in arthritis. J Rheumatol 26:2475-2479

Ginsburg E, Livshits G (1999) Segregation analysis of quantitative traits. Ann Hum Biol 26:103-129 
Gudmundsson H, Gudbjartsson DF, Frigge M et al (2000) Inheritance of human longevity in Iceland. Eur J Hum Genet 8:743-749

Haara MM, Manninen P, Kroger H et al (2003) Osteoarthritis of finger joints in Finns aged 30 or over: prevalence, determinants, and association with mortality. Ann Rheum Dis 62:151-158

Herskind AM, McGue M, Holm NV et al (1996) The heritability of human longevity: a population-based study of 2872 Danish twin pairs born 1870-1900. Hum Genet 97:319-323

Jin SY, Hong SJ, Yang HI et al (2004) Estrogen receptor- $\alpha$ gene haplotype is associated with primary knee osteoarthritis in Korean population. Arthritis Res Ther 6:R415R421

Johansson C, Black D, Johnell O et al (1998) Bone mineral density is a predictor of survival. Calcif Tissue Int 63:190-196

Kadam UT, Jordan K, Croft PR (2004) Clinical comorbidity in patients with osteoarthritis: a case-control study of general practice consulters in England and Wales. Ann Rheum Dis 63:408-414

Kaeberlein M, McVey M, Guarente L (1999) The SIR2/3/4 complex and SIR2 alone promote longevity in Saccharomyces cerevisiae by two different mechanisms. Genes Dev 13:2570-2580

Kalichman L, Cohen Z, Kobyliansky E et al (2002) Interrelationship between bone aging traits and basic anthropometric characteristics. Am J Hum Biol 14:380-390

Kalichman L, Kobyliansky E (2006) Sex- and age-related variations of the somatotype in the Chuvasha population. J Comparat Human Biol HOMO 57:151-162

Kalichman L, Malkin I, Livshits G et al (2006a) Age at Menarche in a Chuvashian Rural Population. Ann Human Biol 33:390-397

Kalichman L, Malkin I, Kobyliansky E (2007a) Time Related trends of menopausal age and reproductive period of women in a chuvashian rural population. Menopause 14:135-140

Kalichman L, Malkin I, Livshits G et al (2007b) Variation of skeletal biomarkers of biological aging in a Chuvashian population: longitudinal study. Am J Hum Biol 19: $74-81$

Kalichman L, Livshits G, Kobyliansky E (2006b) Association between morbidity and skeletal biomarkers of biological aging. Hum Biol 78:77-88

Karasik D, Demissie S, Cupples LA et al (2005) Disentangling the genetic determinants of human aging: biological age as an alternative to the use of survival measures. J Gerontol A Biol Sci Med Sci 60:574-587

Karasik D, Hannan MT, Cupples LA et al (2004) Genetic contribution to biological aging: the Framingham Study. J Gerontol A Biol Sci Med Sci 59:218-226

Karasik D, Otremski I, Barach I et al (1999) Comparative analysis of age prediction by markers of bone change in the hand assessed by roentgenography. Am J Human Biol $11: 31-43$

Kenyon C, Chang J, Gensch E et al (1993) A C. elegans mutant that lives twice as long as wild type. Nature 366:461-464

Kiel DP, Kauppila LI, Cupples LA et al (2001) Bone loss and the progression of abdominal aortic calcification over a
25 year period: the Framingham Heart Study. Calcif Tissue Int 68:271-276

Kirkwood TB, Austad SN (2000) Why do we age? Nature 408:233-238

Kobyliansky E, Livshits G, Pavlovsky O (1995) Population biology of human aging: methods of assessment and sex variation. Hum Biol 67:87-109

Lalouel JM (1983) Segregation analysis of familial data. The mixed model. In: Morton NE, Rao DC, Lalouel JM (eds) Methods in genetic epidemiology. Karger Press, Basel, pp 75-79

Liu YJ, Shen H, Xiao P et al (2006) Molecular genetic studies of gene identification for osteoporosis: a 2004 update. J Bone Miner Res 21:1511-1535

Livshits G, Pavlovsky OM, Kobyliansky E (1996) Population biology of human aging: segregation analysis of bone aging characteristics. Hum Biol 68:539-554

Loughlin J (2005) The genetic epidemiology of human primary osteoarthritis: current status. Expert Rev Mol Med $7: 1-12$

Malkin I, Ginsburg E (2003) Program package for Mendelian analysis of pedigree data (MAN, Version 6). Department of Anatomy and Anthropology, Sackler Faculty of Medicine, Tel Aviv University; Technical Report

McBurney MW, Yang X, Jardine K et al (2003) The mammalian SIR2alpha protein has a role in embryogenesis and gametogenesis. Mol Cell Biol 23:38-54

McGue M, Vaupel JW, Holm N et al (1993) Longevity is moderately heritable in a sample of Danish twins born 1870-1880. J Gerontol 48:B237-244

Mitnitski AB, Graham JE, Mogilner AJ et al (2002) Frailty, fitness and late-life mortality in relation to chronological and biological age. BMC Geriatr 2:1

Morton NE, MacLean CL (1974) Analysis of family resemblance. III. Complex segregation of quantitative traits. Am J Hum Genet 26:489-503

Pavlovsky OM (1987) Biological age of man (in Russian). Moscow University Press, Moscow

Pavlovsky O, Kobyliansky E (1997) Population biology of human aging. Angelo Pontecoroboli Editore Publishing House, Firenze, Italy

Perls TT, Wilmoth J, Levenson R et al (2002) Life-long sustained mortality advantage of siblings of centenarians. Proc Natl Acad Sci USA 99:8442-8447

Plato CC (1987) The effects of aging on bioanthropological variables: changes in bone mineral density with increasing age. Coleg Anthropol 11:59-72

Plato CC, Fox KM, Tobin JD (1994) Skeletal changes in human aging. In: Crews DE, Garruto RM (eds) Biological Anthropology and aging: perspectives on human variation over the life span. Oxford University Press, New York, pp 272-300

Rattan SI (2006) Theories of biological aging: Genes, proteins, and free radicals. Free Radical Research 40: 1230-1238

Remsberg KE, Demerath EW, Schubert CM et al (2005) Early menarche and the development of cardiovascular disease risk factors in adolescent girls: the Fels Longitudinal Study. J Clin Endocrinol Metab 90:2718-2724

Singh R, Kolvraa S, Bross P et al (2006) Reduced heat shock response in human mononuclear cells during aging and its 
association with polymorphisms in HSP70 genes. Cell Stress Chaperones 11:208-215

StatSoft, (2000) Statistica for Windows, Computer program manual. Tulsa, OK

Uitterlinden AG, Burger H, Huang Q et al (1997) Vitamin D receptor genotype is associated with radiographic osteoarthritis at the knee. J Clin Invest 100:259-263
Uttley M, Crawford M (1994) Efficacy of a composite biological age score to predict 10-year survival among Kansas and Nebraska Mennonites. Hum Biol 66:121-144 Yue F, van Meurs BJJ, d'Alesio A et al (2005) Promoter and $3^{\prime}$ untranslated-region haplotypes in the vitamin D receptor gene predispose to osteoporotic fracture: the rotterdam study. Am J Hum Genet 77:807-823 